\title{
Impacto de la caries dental sobre la calidad de vida en preescolares peruanos
}

\author{
Impact of dental caries on their quality of life in preschools peruvians
}

Tania Adriana Cosme Raymundo

\section{RESUMEN}

Objetivo: Determinar el impacto de la caries dental sobre la calidad de vida en preescolares. Materiales y métodos: fue un estudio de tipo observacional, correlacional, transversal y prospectivo en el cual participaron 200 padres de familia, quienes completaron los cuestionarios de ECOHIS; niños evaluados clínicamente utilizando el criterio de diagnóstico ICDAS; y un solo examinador calibrado por un Gold Estándar, presentando un Kappa inter e intraexaminador de 0.79 y 0.81 respectivamente. El análisis estadístico que se utilizó fue Chi cuadrado. Resultados: El impacto de la caries dental en la calidad de vida utilizándose la herramienta de ECOHIS fue considerado medio. El grado de instrucción de los padres no mostró ser una variable que influencie ésta percepción, no encontrándose diferencias estadísticamente significativas $(p=0.53)$. Respecto a la prevalencia de caries dental según el criterio ICDAS fue de $94 \%$ y adaptándolo a la OMS fue de $82 \%$. El c3-6po-d medio de la población fue de 5.05, lo que confirma que la enfermedad estuvo presente en un alto número de los niños de 3 a 5 años evaluados. Conclusiones: A pesar de constatarse una alta prevalencia de caries dental en los niños, dicha afección tuvo un impacto medio en la calidad de vida de los mismos, según la percepción de sus padres de familia.

Palabras claves: Calidad de vida; ICDAS; caries dental; Caries de infancia Temprana. (Fuente: DeCS BIREME)

\section{ABSTRACT}

Objective: To determine the effect of dental caries on the quality of life of pre-school children. Materials and methods: The present was an observational, correlational, cross-sectional and prospective study in which 200 parents participated, who filled the ECOHIS questionnaire. Children were clinically evaluated using the ICDAS diagnostic criteria by a single examiner calibrated by a gold-standard, presenting an inter and intra-examiner Kappa of 0.79 and 0.81 respectively. The statistical analysis used was Chi square. Results: The impact of dental caries on quality of life using the ECOHIS questionnaire was considered intermediate. The parents' educational level did not show to be a variable that influenced their perception, and there were no statistically significant differences $(p=0.53)$. Regarding the prevalence of dental caries according to the ICDAS criterion was $94 \%$ if the WHO criteria is applied $82 \%$ of children showed dental caries. The mean d3-6mf-t of the population was 5.05, confirming that the disease was present in a high number of the examined children aged 3 to 5 years. Conclusions: Despite a high prevalence of dental caries in children, this condition had a medium impact on their Oral Health Related Quality of Life, according to the perception of their parents.

Key words: Quality of life; ICDAS; dental caries; early childhood caries. (Source: MeSH NLM)

Recibido: $\quad 20$ de noviembre de 2018

1 Universidad de San Martin de Porres. Lima, Perú.

${ }^{a}$ Magister

Correspondencia:

Tania Adriana Cosme Raymundo

Correo electrónico: taniacosmer@gmail.com

Aprobado: 24 de enero de 2019

Publicado: 31 de enero de 2019

Este es un artículo Open Access distribuido bajo la licencia Creative Commons Atribución-NoComercialCompartirlgual 4.0

Citar como: Cosme Raymundo T. Impacto de la caries dental sobre la calidad de vida en preescolares peruanos. KIRU. 2019 ; 16(1): 6 -13. https://doi.org/10.24265/kiru.2019.v16n1.01. 


\section{INTRODUCCIÓN}

La calidad de vida tiende a ser un tema de mucho interés en nuestra sociedad, frecuentemente discutido en conferencias de odontología, lo suelen asociar con el impacto de la enfermedad más frecuente como la caries dental y otras alteraciones presentes de la cavidad oral. La literatura actual presenta estudios donde se toma en cuenta la percepción de los padres de familia sobre el estado bucal de sus hijos, para así conocer y brindar una mejor atención sanitaria con el objetivo de conseguir el bienestar de la familia.

Los problemas bucodentales en la población preescolar traen consecuencias en la autoestima del niño, con lo cual se ve afectada su calidad de vida ${ }^{(1,2)}$. El querer darle tratamiento a la enfermedad caries dental ayuda a mejorar el crecimiento del niño, por lo que se recomienda realizar investigaciones más profundas sobre estos temas, para así conseguir prever la enfermedad (3).

La salud bucodental, necesita de continuo monitoreo, por ello debemos identificar cuáles son los grupos de mayor riesgo a contraer lesiones de caries y que poblaciones son las más vulnerables.

A pesar de los estudios realizados, donde se evalúa la prevalencia de caries dental en preescolares, estos son insuficientes, ya que la problemática sigue afectando a gran parte de la población, la cual es sensible a diferentes enfermedades y afecciones que se inician desde muy temprana edad. La prevalencia de caries dental en la dentición decidua fue de $59.1 \%$ según el último reporte del Ministerio de Salud (MINSA) realizado en el $20148^{(4)}$.

La escasa educación bucal de los padres sobre prevención bucal y cuidados bucales; así como también de la mala alimentación de los niños debido a una baja economía o el escaso conocimiento de una dieta equilibrada (no cariogénica), trae como consecuencia lesiones de caries en edades tempranas, teniendo como consecuencia la alta prevalencia, estudios previos observaron en niños menores de 71 meses de edad en zonas urbano marginales de Lima una prevalencia de $62,3 \%{ }^{(5)}$, siendo este un problema que involucra la salud pública, es por ello la necesidad de determinar el impacto de la caries dental sobre la calidad de vida del niño y sus familiares, con la finalidad de plantear estrategias y conciencia en atención odontológica en preescolares.

\section{MATERIALES Y METODOS}

El estudio realizado fue observacional, correlacional transversal, estuvo constituido por 200 niños de 36 a
71 meses de edad con sus respectivos padres de familia ó apoderado. Se realizó en el Colegio Miguel Grau Seminario, ubicado en el Distrito de San Luis, Lima. El tipo de muestreo fue no probabilístico por conveniencia, tomando en cuenta los criterios de inclusión: niños matriculados en el colegio que cuenten con el consentimiento informado debidamente firmado por sus padres o apoderado, padres de familia que vivan con sus hijos participantes en este estudio Los criterios de exclusión fueron: niños con capacidades diferentes, que estén medicados. Para el análisis estadístico se aplicó una estadística descriptiva y la prueba de Chi Cuadrado con la finalidad de asociar variables.

Se hizo un estudio piloto previo, con 20 niños de 12 a 48 meses de edad. Evaluados clínicamente con el criterio de diagnóstico ICDAS y entrevista a sus padres con el cuestionario sobre la escala de medición del Impacto de la Salud Oral (Early Childhood Oral Health Impact Scale) ECOHIS, en el centro educativo inicial $N^{\circ} 157$ del Distrito de Cieneguilla, en la cual se verificó la efectividad de las fichas de recolección de datos y la metodología a emplear.

El trabajo de campo tuvo dos partes: una entrevista con el cuestionario ECOHIS, dirigida a los padres de familia o apoderados y la evaluación clínico visual de las lesiones cariosas en preescolares.

Para la entrevista se empleó la versión en español, validada para Perú (7). El ECOHIS es un cuestionario que consta de 13 ítems que evalúa el impacto en el niño; dividido en 6 dominios: síntomas del niño (1 ítem), funciones del niño (4 ítems), psicología del niño (2 ítems), autoimagen/interacción social del niño (2 ítems) y el impacto en los padres de familia o apoderado considerando el estrés de los padres (2 ítems) y funciones de la familia (2 ítems). Los cuestionarios fueron llenados por los padres de familia a la hora de salida del colegio, contando con un tiempo prudencial y constante apoyo en caso surgieran dudas.

La evaluación clínico visual de las lesiones cariosas en preescolares, fue realizada por una sola examinadora entrenada y calibrada en ICDAS, cuyo coeficiente de Kappa intra examinador fue 0.79 e inter examinador de 0.81, calibración realizada por una Gold Estándar certificada por (International Association Dental Reserch) IADR. Es importante recalcar que este sistema de evaluación diagnostica la enfermedad desde sus estadios iniciales (manchas blancas). Se realizó el protocolo estricto de ICDAS(6) empezando por el cepillado dental, uso de dentífrico y hilo dental, con la finalidad de evaluar lesiones iniciales de caries dental, se secó cada pieza dentaria con gasa, examinando cada superficie, por cuadrantes de forma 
ordenada (mesial, oclusal, distal, bucal y lingual), empezando por el cuadrante cinco y terminando en el cuadrante 8 (55 -65, 75 -85), Se utilizó Fronto luz (blanca) Energizer (30 lumens), Se adaptó una mesa como camilla, con colchoneta aislada con material plástico (film) tomando todas las medidas de bioseguridad pertinentes, para tener mejor visión del paciente, donde el examinador se ubicó por detrás del mismo en posición de doce horas, según las agujas del reloj, Los datos fueron registrados con una grabadora, para luego ser anotados en las fichas de evaluación ICDAS - USMP.

A todos los niños se les entregó su cepillo dental y pasta dental al finalizar el examen clínico. Se empleó el criterio de diagnóstico ICDAS, que cuenta con códigos que van de 0 a 6 , uniendo los códigos 1 y 2 por tratarse de un estudio epidemiológico.

\section{RESULTADOS}

Fueron 200 niños evaluados de los cuales 108 eran del sexo femenino y 92 del sexo masculino, en cuanto a la edad, se trabajó con tres grupos etarios: 46 niños (36 a 48 meses), 76 niños (48 a 59 meses) y 78 niños (60 a 71 meses) como se presenta en la tabla 1. Cabe señalar que las molares deciduas fueron las más afectadas por lesiones de caries dental en la superficie bucal y oclusal de ambas arcadas.

Tabla 1. Distribución muestral por edad y sexo en niños de 36 a 71 meses de edad del Colegio Miguel Grau Seminario, Distrito de San Luis - Lima, 2017.

\begin{tabular}{|c|c|c|c|c|c|c|c|c|}
\hline \multirow{3}{*}{ SEXO } & \multicolumn{6}{|c|}{$\begin{array}{l}\text { EDADES } \\
\text { (meses) }\end{array}$} & & \\
\hline & \multicolumn{2}{|c|}{36 a 47} & \multicolumn{2}{|c|}{48 a 59} & \multicolumn{2}{|c|}{60 a 71} & \multicolumn{2}{|c|}{ TOTAL } \\
\hline & $\mathbf{N}$ & $\%$ & $\mathbf{N}$ & $\%$ & $\mathbf{N}$ & $\%$ & $\mathbf{N}$ & $\%$ \\
\hline$F$ & 29 & 63.0 & 37 & 48.7 & 42 & 53.8 & 108 & 54.0 \\
\hline $\mathbf{M}$ & 17 & 37.0 & 39 & 51.3 & 36 & 46.2 & 92 & 46.0 \\
\hline TOTAL & 46 & 100.0 & 76 & 100.0 & 78 & 100.0 & 200 & 100.0 \\
\hline
\end{tabular}

En la tabla 2, se observa en el corte c2-6-s que incluye lesiones no cavitadas (manchas blancas) una prevalencia de 94\% y en el corte c3-6 considerando lesiones cavitadas, adaptándolo al índice cpo-d según la OMS de $82 \%$ obteniendo una diferencia de $12 \%$, el cual no sería apreciable si solo se utiliza el índice del cpo donde consideran solo lesiones cavitadas.

Tabla 2. Prevalencia de caries dental, considerando lesiones no cavitadas y lesiones cavitadas en niños de 36 a 71 meses de edad del Colegio Miguel Grau Seminario, Distrito de San Luis - Lima, 2017

\begin{tabular}{cccccc}
\hline $\begin{array}{c}\text { EDAD } \\
\text { (meses) }\end{array}$ & N Niños & $\begin{array}{c}\text { c2-6-s } \\
\%\end{array}$ & $\begin{array}{c}\text { c3-6-s } \\
\%\end{array}$ & $\begin{array}{c}\text { c4-6-s } \\
\%\end{array}$ & $\begin{array}{c}\text { c5-6-s } \\
\%\end{array}$ \\
\hline $\mathbf{3 6}$ a 47 & 46 & 93.5 & 80.4 & 63.0 & 54.3 \\
$\mathbf{4 8}$ a 59 & 76 & 97.4 & 88.2 & 68.4 & 56.6 \\
$\mathbf{6 0}$ a 71 & 78 & 91.0 & 76.9 & 59.0 & 53.8 \\
TOTAL & 200 & 94.0 & 82.0 & 63.5 & 55.0 \\
\hline
\end{tabular}


En la tabla 3 se observa la contrastación de las variables: grado de instrucción de los padres de familia y presencia / ausencia de caries dental en los niños, al aplicar la Prueba del Chi cuadrado de no se reportó asociación, ya que el valor fue de $p=0.53$. (Tabla 3 )

Tabla 3. Relación entre el grado de instrucción de los padres y presencia de caries dental en niños de 36 a 71 meses del Colegio Miguel Grau Seminario, Distrito de San Luis - Lima, 2017.

\begin{tabular}{lcccc}
\hline $\begin{array}{c}\text { GRADO DE INSTRUCCIÓN DE LOS } \\
\text { PADRES DE FAMILIA / } \\
\text { APODERADO }\end{array}$ & \multicolumn{3}{c}{ CARIES DENTAL } & TOTAL \\
\hline \multirow{2}{*}{ Sin escolaridad } & $\mathrm{N}$ & SI & NO & \\
& $\%$ & $2,1 \%$ & $0,0 \%$ & $2,0 \%$ \\
Primaria & $\mathrm{N}$ & 21 & 0 & 21 \\
& $\%$ & $11,2 \%$ & $0,0 \%$ & $10,5 \%$ \\
Secundaria & $\mathrm{N}$ & 145 & 10 & 155 \\
& $\%$ & $\mathbf{7 7 , 1} \%$ & $\mathbf{8 3 , 3} \%$ & $77,5 \%$ \\
Superior & $\mathrm{N}$ & 18 & 2 & 20 \\
& $\%$ & $9,6 \%$ & $16,7 \%$ & $10,0 \%$ \\
TOTAL & $\mathrm{N}$ & 188 & 12 & 200 \\
\hline
\end{tabular}

Al evaluar a los padres de familia con el cuestionario ECOHIS se reportó con picos altos al impacto en el niño, los ítems sobre: dificultad para pronunciar alguna palabra y problemas para dormir, seguidas de dificultad para tomar bebidas calientes o frías y ha evitado sonreír. Respecto al impacto en el padre de familia o apoderado fue la preocupación por el estado de salud bucal de su hijo (Figura 1).

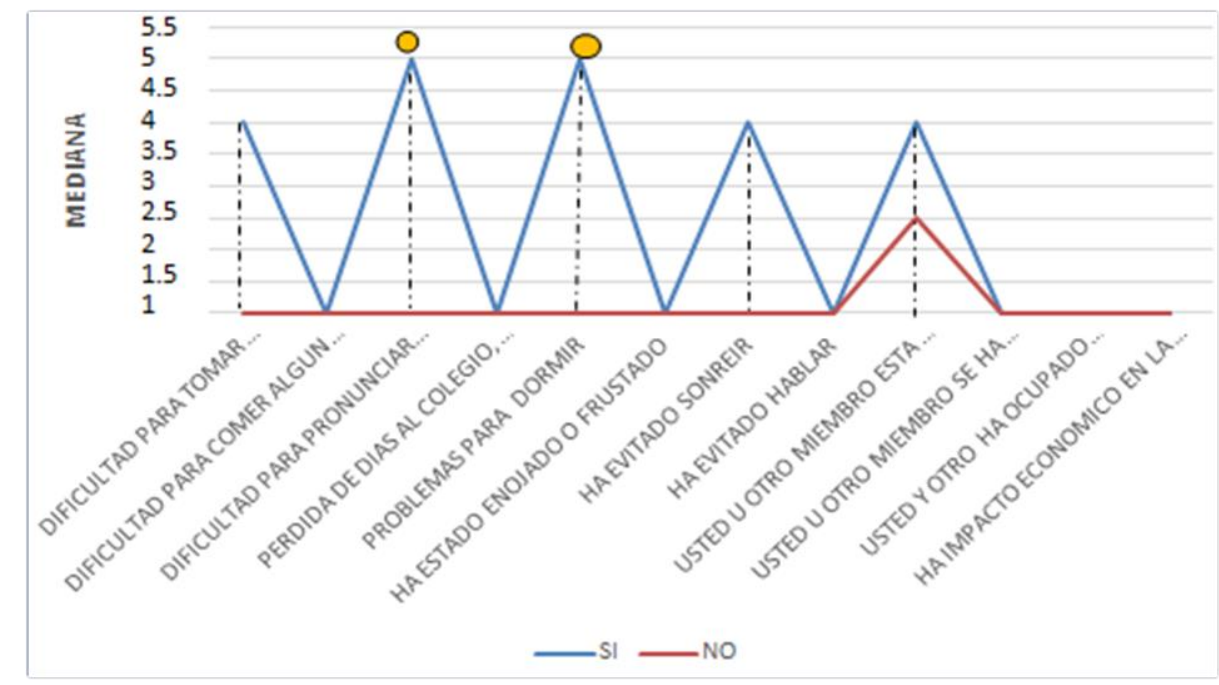

Figura 1: Percepción del padre de familia / apoderado sobre la calidad de vida relacionada a la salud bucal en niños de 36 a 71 meses del Colegio Miguel Grau Seminario, Distrito de San Luis - Lima, 2017 
En cuanto a la relación del impacto de la caries dental sobre la calidad de vida en los niños de 36 a 71 meses, se determinó que si existe un impacto medio sobre la
Calidad de vida, lo cual se corroboró con la prueba de Chi cuadrado, siendo el valor de $\mathrm{p}<0.05$ mostrando significancia y asociación (Tabla 4).

Tabla 4. Relación de la caries dental por superficie sobre la calidad de vida en niños de 36 a 71 meses del Colegio Miguel Grau Seminario, Distrito de San Luis - Lima, 2017.

\begin{tabular}{|c|c|c|c|c|c|}
\hline & & & \multicolumn{2}{|c|}{$\begin{array}{l}\text { CARIES DENTAL } \\
\text { PREESCOLARES }\end{array}$} & \multirow[b]{2}{*}{ TOTAL } \\
\hline \multicolumn{3}{|c|}{ PADRES DE FAMILIA / APODERADO } & SI & NO & \\
\hline \multirow[t]{6}{*}{ CALIDAD DE VIDA } & \multirow{2}{*}{ BAJO } & $n$ & 75 & 0 & 75 \\
\hline & & $\%$ & $37.5 \%$ & $0.0 \%$ & $37.5 \%$ \\
\hline & \multirow{2}{*}{ MEDIO } & $\mathrm{n}$ & 95 & 2 & 97 \\
\hline & & $\%$ & $47.5 \%$ & $1.0 \%$ & $48.5 \%$ \\
\hline & \multirow{2}{*}{ ALTO } & $\mathrm{n}$ & 18 & 10 & 28 \\
\hline & & $\%$ & $9.0 \%$ & $5.0 \%$ & $14.0 \%$ \\
\hline \multirow{2}{*}{\multicolumn{2}{|c|}{ TOTAL }} & $\mathbf{n}$ & 188 & 12 & 200 \\
\hline & & $\%$ & $94.0 \%$ & $6.0 \%$ & $100.0 \%$ \\
\hline
\end{tabular}

\section{DISCUSIÓN}

La alta prevalencia de caries dental en preescolares, sigue afectando a la población peruana, la cual no es indiferente para la sociedad. Comprender todas las consecuencias que trae consigo la falta de atención en los niños debe conducir a lograr diagnosticar y detener la enfermedad desde edades tempranas, ya sea mediante métodos preventivos - promocionales e interceptivos, con la finalidad de evitar tratamientos complejos a futuro y consecuencias que no solo conlleven alteraciones en la salud bucal, si no en la salud general y en la calidad de vida.

El impacto de la enfermedad caries dental sobre la calidad de vida en preescolares, puede ser identificado por sintomatología dolorosa, inapetencia, ausencia escolar, alteraciones en el sueño, alteraciones al masticar, que el niño evite sonreír afectando la autoestima, así como también, ejerciendo un impacto en la vida de sus familiares que invierten tiempo, preocupaciones, gastos, ausentismo laboral, entre otros. Las lesiones cariosas no tratadas seguirán su curso de evolución generando alteraciones en la calidad de vida negativamente, es por ello que la percepción de los padres de familia o apoderados es determinante.

En el presente estudio encontró una prevalencia de $94 \%$ considerando las lesiones no cavitadas (manchas blancas) similar a los resultados obtenidos por López $R$ et al $(76,47 \%)^{(8)}$, Villena RS et al $(86.9 \%)^{(4)}$, Alvitez $\mathrm{P}(94.4 \%)^{(9)}$, Merchán G $(100 \%)^{(10)}$.
Muchos autores no suelen emplear el criterio de diagnóstico ICDAS en sus investigaciones; pero se puede realizar comparaciones desde el c $3-6$ pod (lesiones cavitadas) al tomar como referencia el componente "c" del criterio del cpo-d, cuya prevalencia fue de $82 \%$ la cual sigue siendo alta. Las lesiones iniciales suelen pasar desapercibidas por los padres de familia y no le dan la importancia que deberían, lo ideal sería detectar tempranamente la enfermedad y detenerla desde su primer signo clínico, con la finalidad de obtener datos que incluyan la presencia de la enfermedad desde el primer signo clínico. Por ello es importante hacer énfasis en desarrollar estudios de investigación con el criterio de diagnostico ICDAS por ser más preciso en la detección de lesiones cariosas iniciales.

Se encontró en el presente estudio que la prevalencia de las lesiones cariosas coincidió con los resultados obtenidos por Merchán G. ${ }^{(10)}$ Con relación a la media de caries dental en preescolares, al utilizar el criterio de diagnóstico ICDAS asemejándolo al cpo-d de la OMS, fueron similares: para el corte c2-6po-d y c36 po-d en los tres grupos etarios, evidenciando que las lesiones cariosas afectaban un rango de 17 piezas dentarias, lo que sigue certificando que los niños evaluados presentaban una alta prevalencia. Las superficies dentarias según el criterio de diagnóstico ICDAS de las lesiones cariosas cavitadas y no cavitadas en ambas arcadas, se encontraron que la superficie bucal y oclusal de las segundas molares deciduas superiores e inferiores, considerando una 
media por superficie en el corte de c2-6-s fue de 8.77, siendo las lesiones no cavitadas (manchas blancas) las más prevalentes en las edades estudiadas, coincidiendo con lo hallado por Alvitez P. (9), Merchán $\mathrm{G}^{(10)}$ y Villena RS et $\mathrm{a}^{(4)}$, es importante recalcar que las molares superiores $e$ inferiores presentaron lesiones no cavitadas (manchas blancas) en fosas y fisuras en la superficie oclusal, debido a su anatomía sinuosa siendo estas muy profundas y retentivas, la cuales predisponen para la instalación de la enfermedad. Se encontró también alto porcentaje en la superficie mesial de los incisivos centrales superiores al ser evaluados con el índice cpo-s, el cual fue similar utilizando el criterio diagnóstico ICDAS, esto se debe posiblemente a la falta de espacios inter proximales y presencia de arco tipo II de Baume en dicha arcada.

Con relación al impacto de la calidad de vida, muchos Investigadores como Raymundo de Andrade $\mathrm{L}$. et $a l^{(11)}$, Bonecker M. et $a l^{(12)}$ Leal S. et al(13), Scarpelli A. et $a^{(14)}$, Kramer $P$. et $a l^{(15)}$, Correa- Faria $P$ et $a^{(16)}$, Tello G. et al(17) y Guedes R. et al ${ }^{(18)}$ demostraron que el bienestar de los niños podría ser perjudicado por problemas bucales, como la presencia de la enfermedad caries dental, causando un impacto negativo en su calidad de vida, alterando su autoestima, repercutiendo su salud general, sin embargo el estudio realizado por el investigador Piovesan C. et $a^{\left({ }^{(19)}\right.}$ sobre la actividad de la lesión cariosa así como la severidad, utilizando el criterio de diagnóstico ICDAS, solo hasta el código 4, hallaron un bajo impacto en la calidad de vida de los niños.

Se podría considerar que al no ser evaluadas las lesiones cavitadas código 5 y 6 de ICDAS, no obtuvieron manifestación clínica visiblemente complejas lo que indica que para la percepción de sus padres no fue perjudicial en su calidad de vida. Respecto a la percepción de responsabilidad de los padres por la presencia de caries dental, Cesarino $\mathrm{M}$. et $\mathrm{al}^{(20)}$, hallaron que esta aumenta con la aparición avanzada de la enfermedad, lo cual conlleva a necesitar tratamientos urgentes, como también lo refiere Vieira - Andrade R. et $a^{(21)}$, Wong S. et $a^{(22)} y$ Correa - Faria P. et $a^{(23)}$, sobre las consecuencias de caries no tratadas, que presentaron impacto negativo en preescolares, sobre la percepción de responsabilidad y preocupación de los padres o cuidador Arrow P.(24) encontró ausencia de responsabilidad y poca importancia en el cuidado de los dientes deciduos, así como también un desconocimiento sobre tratamientos preventivos, resultado que no coincide con los obtenidos en este estudio ya que al realizar la evaluación del ECOHIS hubieron padres que manifestaron estar preocupados por la salud bucal de su hijo, aunque al realizar el examen clínico no se corroboró dicha aseveración, ya que habían muchos niños enfermos.
La auto percepción puede variar según las creencias de cada individuo, según los conceptos personales que tienen sobre salud y enfermedad. En el presente estudio con relación a la percepción del impacto sobre la calidad de vida, presentó un impacto medio, según la percepción de los padres de familia / apoderado, mostrando una tendencia alta y baja en las respuestas del cuestionario ECOHIS, cuyas calificaciones medianas de calidad sobresalen en el grupo con caries dental llamando la atención los valores altos en "dificultad para pronunciar algunas palabras" "problemas para dormir", seguidas de "dificultad para tomar bebidas calientes", "evitar sonreír". En el caso de la familia sobre que "está preocupado por el niño", demostrando así que si existe significancia con relación a la presencia de caries dental.

Autores como Alves $\mathrm{H}$. et $a^{(25)}$, Guedes RS. et $a^{(26)}$, Guedes R. et a ${ }^{(27)}$ y Díaz S. et $a^{(28)}$. también hallaron baja percepción de los padres, a pesar de que habían niños que presentaban sintomatología dolorosa a causa de lesiones cariosas, sin embargo la presencia de estas, no afectaron su calidad de vida, muchas veces los padres de familia no tienen la misma percepción que sus hijos y pueden pasar desapercibidos muchos signos y síntomas de la enfermedad, avalando que solo son dientes deciduos que pronto serán exfoliados y por lo tanto no necesitan ser restaurados 0 textualmente "No amerita tratamiento", siendo necesario concientizar y educar sobre prevención e importancia de la dentición decidua, lo que puede afectar una lesión de caries no tratada, puede repercutir en el estado general de su niño y también en los gastos económicos que le demandaría a la familia, por lo que se hace la siguiente pregunta: ¿Por qué esperar tanto? Se debe evitar daños mayores a los niños, la enfermedad de caries dental es prevenible.

Con relación al nivel de grado de instrucción de los padres autores como Castro F. et $a^{(29)}$, Pinto Sarmiento A. et $a^{(30)}$, Nanayakkara V. et $a^{(31)} y$ Chaffee B. et a/(32) manifestaron que la falta de educación de los padres de familia puede intervenir en la percepción sobre la salud oral de sus hijos, encontraron asociación entre el bajo nivel socioeconómico, el grado de instrucción de los padres, con la presencia de caries dental lo cual presentó impacto negativo, lo que no concuerda con los resultados de este estudio, podría ser que la percepción de la calidad de vida puede ser cambiante en los distintos grupos socioeconómicos.

Finalmente se concluye que la caries de infancia temprana permite enfocarse en métodos preventivos abordándolos de forma paulatina y motivando a los padres de familia, empezando con educación desde casa, además de poder trabajar desde los centros educativos iniciales para poder modificar la percepción 
de los padres y a sus profesores, proporcionando diferentes alternativas hasta encontrar la más apropiada para el grupo poblacional estudiado con el apoyo de cuestionarios validados para la primera infancia y la etapa escolar como recomienda Zaror C. et al ${ }^{(33)}$. EL trabajo de controlar y vigilar los factores causales de la enfermedad sigue siendo un reto en esta sociedad, cuyo objetivo es conseguir calidad de vida en toda la población, especialmente en este grupo etario donde inician su autovaloración personal.

\section{Se llegó a las siguientes conclusiones:}

- La prevalencia de lesiones no cavitadas en los niños de 36 a 71 fue alta (94\%), no detectándose diferencia estadísticamente significativa en ambos sexos. Al excluirse las lesiones de caries no cavitadas y adaptar los niveles a c3-6po-d (OMS), la prevalencia también fue considerada alta $82 \%$, sin embargo, una diferencia de $12 \%$ de lesiones no detectadas con este corte son reportadas. No se encontraron diferencia estadísticamente significativa en ambos sexos. La prevalencia de piezas dentales perdidas por caries y obturadas, fue considerada baja, equivalente a $4 \%$ y $6 \%$ respectivamente. Una mayor prevalencia de caries fue evidente en las superficies bucales y oclusales de las segundas molares superiores e inferiores.

- El valor medio del cpo-d y cpo-s incluyendo lesiones cavitadas y no cavitadas fue 5.05 y 8.77 respectivamente.

- Según la percepción de los padres de familia, la dificultad para pronunciar y problemas para dormir de los niños fueron los picos más altos de prevalencia en cuanto al ECOHIS.

- Respecto al grado de instrucción de los padres de familia / apoderados y la relación con la presencia de caries dental en los niños, no presentó significancia estadística, valor de $\mathrm{p}=0,53$.

- Hubo relación entre la caries dental y la calidad de vida, a pesar de constatarse una alta prevalencia de caries dental en los niños, dicha afección tuvo un impacto medio en la calidad de vida de los mismos, según la percepción de sus padres de familia.

Contribuciones de autoría: TCR diseño del estudio, recopilo y analizó los datos. Redacto y aprobó el artículo.

Fuente de financiamiento: El estudio fue financiado por el autor.

Conflicto de intereses: La autora declaro no tener conflictos de interés.

\section{REFERENCIAS BIBLIOGRAFICAS}

1. Alkarimi $H A$, Watt $R G$, Pikhart $H$, Jawadi $A H$, Sheiham A, Tsakos G. Impact of treating dental caries on schoolchildren's anthropometric dental satisfaction and appetite outcomes: a randomized controlled trial. BMC Public Health. 2012; 12(1): 706.

2. Anderson $\mathrm{H}$, Drummond B, Thomson W. Changes in aspects of children's oral-health-related quality of life following dental treatment under general anesthesia. J Pediatr Dent. The British Paedodontic Society and the International Association of Dentistry for Children. 2004; 14(5): 317-325.

3. Monse B, Sheiham A, Grijalva - Eternod C, Van Palenstein Helderman W, Hobdell M. The effects of extraction of pulpally involved primary teeth on weight and BMI in underweight Filipino children. A cluster randomized clinical trial. BMC Public Health. 2012; 12(1): 725.

4. Ministerio de Salud. Guía de práctica clínica para la prevención, diagnostic y tratamiento de la caries dental en niños y niñas, Perú; 2017. Disponible en: http://bvs.minsa. gob.pe/local/MINSA/4195.pdf

5. Villena RS, Pachas F, Sánchez Y, Carrasco M. Prevalencia de Caries en Infancia Temprana en niños menores de 6 años de edad. Residentes en población urbana marginal de Lima Norte: Rev. Estomatol Hered.. 2011; 21(2): 79 -86.

6. Ismail A, Sohn W, Tellez M, Amaya A, Sen A, Pitts $\mathrm{N}$, et al. The International Caries Detection and Assessment System (ICDAS) an integrated system for measuring dental caries. Community Dent. Oral Epidemiol. 2007; 35(3): 170-178.

7. Bordoni N, Claravino O, Zambrano O, Villena R, E. Beltrán Aguilar E. Early Childhood Oral Health Impact Scale (ECOHIS). Act Odont Latinoam. 2012; 25: 270-278.

8. López R, García C. Calidad de vida y problemas bucales en preescolares de la Provincia de Huaura, Lima. Rev. Estomatol Herediana. 2014; 23(3), 139-147.

9. Alvitez P. Prevalencia de caries de Infancia temprana según el criterio de diagnóstico ICDAS en niños de 12 a 71 meses residentes en el cercado de Lima. [Tesis para optar la especialidad de odontopediatría] Universidad San Martín de Porres, 2015.

10. Merchan G. Prevalencia de caries de Infancia temprana según el criterio de diagnóstico ICDAS en niños de 12 a 71 meses residentes del Distrito de Santa Anita, Lima-Perú. [Tesis para optar la especialidad de odontopediatría] Universidad San Martín de Porres, 2015.

11. Raymundo de Andrade L. Buczynski, Raggio A, Castro G, Ribeiro de Souza I. Impacto de la salud oral en la calidad de vida en niños pre escolares, Percepción de los responsables. Act Odontol Venezolana. 2011; 49(4): 1-9.

12. Bönocker M, Abanto J, Tello G, Butini L. Impact of caries on preschool childrens quality of life: an update. Pediatr Dent. 2012; 26 (Spcc Iss 1): 103 107.

13. Leal SC, Bronkhorst EM, Fan M, Frencken JE. Untreated cavitated dentine lesions: Impact $n$ Childrens quality of life. Caries Res. 2012; 46: 102 $-106$. 
14. Scarpelli AC, Paiva SM, Viegas CM, Cruz A, Ferreira FM, Pordeus IA. Oral health - related quality of life among Brazilian preschool children. Community Dent Oral Epidemiol. 2013; 41: 336344.

15. Kramer P, Feldens C, Helena Ferreira S, Bervian $\mathrm{J}$, Rodrigues $\mathrm{P}$, Peres $\mathrm{M}$. Exploring the impact of oral diseases and disorders on quality of life of preschool children. Community Dent Oral Epidemiol. 2013; 41(4): 327-335.

16. Correa - Faria P, Paixao - Goncalves S, Martins Paiva S, Martins-Junior P, Vieira - Andrade R, Silva Marques L, Ramos - Jorge M. Dental caries, but not malocclusion or developmental defects, negatively impacts preschoolers quality of life. Int $\mathrm{J}$ Paediatr Dent. 2015; DOI: 10.1111/IPD.12190: 19.

17. Tello G, Abanto J, Butini L, Murakani C, Bonini G, Bönecker M. Impacto de los principales problemas de salud bucal en la calidad de vida de preescolares. Rev "ODONTOLOGÍA". 2016; 19(2): $42-52$.

18. Guedes RS. Ardenghi TM, Piovesan C, Emmanuelli B, Mendes FM. Influence of initial caries lesions on quality of life in preschool children: a 2-year cohort study. Community Dent Oral Epidemiol. 2016; 44: $292-300$.

19. Piovesan C, Ardenghi TM, Guedes RS, Ekstrand $\mathrm{KR}$, Braga MM, Mendes FM. Activity assessment has Little impacto $n$ caries parameters reduction in epidemiological surveys with preschool children. Community Dent Oral Epidemiol. 2012; DOI: 10.1111: 1-7.

20. Cesarino Gomes M, Pinto- Sarmiento $T$, Clementino M, Martins C. Association between parental guilt and oral health problems in preschool children: A hierarchical approach. 2014; DOI: 10.1186/1471-2458-14-854: 1 - 16.

21. Vieira - Andrade R, Martins- Júnior $P$, CorreaFaria P, Silva Marques L, Paiva Martins S, RamosJorge M. Impact of oral mucosal conditions on oral health-related quality of life in preschool children: a hierarchical approach. Int J Paediatr Dent. 2014; DOI: 10.1111/ipd.12107: 1 - 10 .

22. Wong S, Anthonappa R, Ekambaram M, Colman McG, Nigel K, Winters J. Quality of life changes in children following emergecy dental extractions under general anaesthesia. Int $\mathrm{J}$ Paediatr Dent. 2016; DOI: 10.1111/ipd.12241: $80-86$.

23. Correa - Faria P, Daher A, Freire MDCM, De Abreu MHNG, Bonecker M, Costa LR. Impact of untreated dental caries severity on the quality of life of preschool children and their families: a cross- sectional study. Qual Life Res. 2018; DOI: 10.1007/s11136-018-1966-5.

24. Arrow Peter. Responsiveness and sensitivity of the Early Childhood Oral Health Impact Scale to primary dental care for early childhood caries. Community Dent Oral Epidemiol. 2015; 44: 1-10.

25. Alves Clementino M, Cesarino Gomes M, PintoSarmiento T, Castro Martins C, Granville- García A, Martins Paiva S. Perceived Impact of Dental Pain on the Quality of life of Preschool Children and Their Families. PLoS One. 2015; DOI: 10.1371/J.Pone.0130602: 1 - 13.

26. Guedes RS, Piovesan C, Antunes J, Mendes F, Ardenghi T. Assessing individual and neighborhood social factors in child oral healthrelated quality of life: a multilevel analysis. Qual Life Research, 2014; 23(9): 2521-2530.

27. Guedes RS, Ardenghi T, Emmanuello B, Piovesan Ch, Mendes F. Sensitivity of an oral health related quality of life questionnaire in detecting oral health impairment in preschool children. IAPD 2017; DOI: 10.1111/ipd.12328: 1- 10.

28. Diaz Sh, Abanto J. Crespo Z, Villafanë C, Escorcia E. Actitudes de padres sobre salud bucal, creencias sobre dentición decidua y su asociación con caries dental y calidad de vida en la salud bucal en preescolares. [Tesis para Especialista en Odontopediatría] Universidad de Cartagena. 2016.

29. Castro F, Raggio D, Imparato JC, Piovesan Ch, Bonini G. Impacto dos Problemas Bucais na Qualidade de Vida em Pré-Escolares. Pesq Bras Odontoped Clin Integr. 2013; 13(4):361- 369.

30. Pinto-Sarmento T, Abreu M, Gomes M, Costa E, Martins C, Paiva S, et al. Determinant Factors of Untreated Dental Caries and Lesion Activity in Preschool Children Using ICDAS. 2016; 11(2): 111.

31. Nanayakkara V, Renzaho A, Oldenburg B, Ekanayake L. Ethnic and socio - economic disparities in oral health outcomes and quality of life among Sri Lankan preschoolers: a cross sectional study. Inter J Equity Health. 2013; 12(89): 1- 9 .

32. Chaffee $B$, Rodrigues $\mathrm{P}$, Kramer $\mathrm{P}$, Vitolo $\mathrm{M}$, Feldens $C$. Oral health - related quality - of- life scores differ by socieconomic status and caries experience. Community Dent Oral Epidemiol. 2017; DOI: 10.1111/cdoe.12279.

33. Zaror C, Pardo Y, Espinoza- Espinoza G, Pont A, Muños- Millán P, Martins- Zapata M, Vilagut G, Forero C, Garin O, Alonso J, Ferrer M. Clinical Oral Investigations. 2018; DOI: org/10.1007/s00784018-2406-1: 1 - 15 . 